\title{
A double detached shell around a post-red supergiant: IRAS 17163-3907, the Fried Egg nebula ${ }^{\star \star \star \star}$
}

\author{
E. Lagadec ${ }^{1}$, A. A. Zijlstra ${ }^{2}$, R. D. Oudmaijer ${ }^{3}$, T. Verhoelst ${ }^{4}$, N. L. J. Cox ${ }^{4}$, \\ R. Szczerba ${ }^{5}$, D. Mékarnia ${ }^{6}$, and H. van Winckel $^{4}$
}

\author{
${ }^{1}$ European Southern Observatory, Karl Schwarzschild Strasse 2, 85748 Garching, Germany \\ e-mail: elagadec@eso.org \\ 2 Jodrell Bank Center For Astrophysics, The University of Manchester, School of Physics \& Astronomy, Manchester M13 9PL, UK \\ 3 School of Physics and Astronomy, University of Leeds, Leeds LS2 9JT, UK \\ ${ }^{4}$ Instituut voor Sterrenkunde, K.U. Leuven, Celestijnenlaan, 200D, 3001 Leuven, Belgium \\ 5 N. Copernicus Astronomical Center, Rabiańska 8, 87-100 Torùn, Poland \\ ${ }^{6}$ Lab. H. Fizeau, CNRS UMR 6525, Univ. de Nice-Sophia Antipolis, Observatoire de la Côte d'Azur, 06108 Nice, France \\ Received 20 June 2011 / Accepted 27 September 2011
}

\section{ABSTRACT}

\begin{abstract}
Context. We performed a mid-infrared imaging survey of evolved stars to study the dust distribution in circumstellar envelopes around these objects and to understand the mass-loss mechanism responsible for the formation of these envelopes better. During this survey, we resolved the circumstellar environment of IRAS 17163-3907 for the first time (hereafter IRAS 17163), which is one of the brightest objects in the mid-infrared sky, but is surprisingly not well studied.

Aims. Our aim is to determine the evolutionary status of IRAS 17163 and study its circumstellar environment to understand its massloss history.

Methods. We obtained diffraction-limited images of IRAS 17163 in the mid-infrared using VISIR on the VLT. Optical spectra of the object allowed us to determine its spectral type and estimate its distance through diffuse interstellar bands.

Results. We show that IRAS 17163 is a post-red supergiant, possibly belonging to the rare class of yellow hypergiants, and is very similar to the well-studied object IRC +10420. Our mid-infrared images of IRAS 17163 are the first direct images of this bright midinfrared source. These images clearly show a double dusty detached shell around the central star, caused by successive ejections of material on a timescale of the order of 400 years and a total circumstellar mass exceeding than $4 M_{\odot}$. This indicates that non-quiescent mass-loss occurs during this phase of stellar evolution.
\end{abstract}

Key words. circumstellar matter - stars: mass-loss - supergiants - infrared: stars

\section{Introduction}

After exhausting the hydrogen in their core at the end of the main sequence, stars with initial masses in the range 8-40 $M_{\odot}$ become large and cool red supergiants. When they leave the red supergiant branch, those stars evolve in the yellow hypergiant phase, followed by the luminous blue variable (LBV) phase, to finally become Wolf-Rayet stars (Oudmaijer et al. 2009). The stellar winds associated with these phases, followed by a final supernova explosion, are essential for the chemical enrichment of galaxies and provide kinetic energy that can trigger star formation. These stellar winds are likely caused by a combination of pulsation and radiation pressure on dust. This leads to the formation of dusty circumstellar envelope that makes post-mainsequence objects bright infrared sources.

As part of a mid-infrared imaging survey of evolved stars (Lagadec et al. 2011), we observed the very bright mid-infrared source (with a $12 \mu \mathrm{m}$ flux $F_{12}=1243$ Jy) IRAS $17163-3907$ (hereafter IRAS 17163).

* Based on observations made with the Very Large Telescope at Paranal Observatory under program 081.D-0130(A).

$\star \star$ Based on observations made with the Mercator Telescope, operated on the island of La Palma by the Flemish Community, at the Spanish Observatorio del Roque de los Muchachos of the Instituto de Astrofsica de Canarias.
IRAS 17163 (a.k.a. Hen 1379) was discovered by Henize in 1976 during a survey of emission line stars in the southern sky. From their $2.2 \mu \mathrm{m}$ mapping (Valinhos 2.2 micron survey), Epchtein et al. (1987) classified this object as a pre-planetary nebula (PPN) candidate. Optical spectra of this object allowed us to show that the distance to this object is four times greater than previously assumed, and thus too bright to be a post-asymptotic giant branch (AGB) star.

We report here the direct detection of a double detached shell around the central star of this post-red supergiant object.

\section{Observations and data reduction}

We observed IRAS 17163 in the mid-infrared with VISIR on the VLT (Lagage et al. 2004) with three filters: PAH1 $(8.59 \mu \mathrm{m}$, half bandwidth $0.42 \mu \mathrm{m}), \operatorname{SiC}(11.85 \mu \mathrm{m}, 2.34 \mu \mathrm{m})$ and NeII $(12.81 \mu \mathrm{m}, 0.21 \mu \mathrm{m})$. We used the imager in burst mode, using a pixel scale of 0.075 arcsec and a field of view of $19.2 \times 19.2$ arcsec. With the burst mode, chopping and nodding images are recorded, which allows the reconstruction of quality-enhanced images using shift-and-add techniques. We used the standard chopping/nodding technique to remove the background. We shifted and added the images using a maximum of correlation algorithm, after removing the bad images. Our observations were obtained during one of the driest nights ever in Paranal $(0.43 \mathrm{~mm}$ 

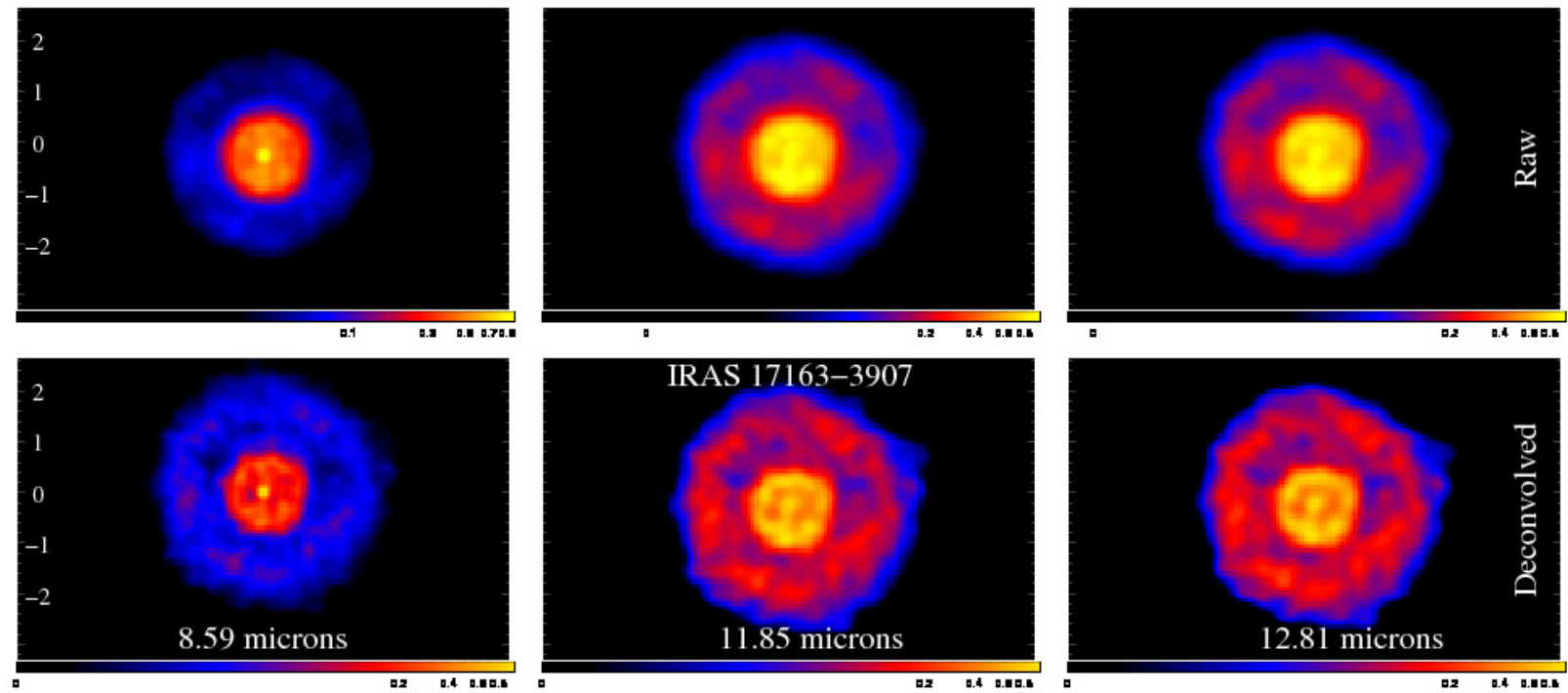

Fig. 1. VISIR/VLT mid-infrared images of IRAS 17163 at different wavelengths. The raw and deconvolved images are displayed for each filter. North is up and East left. The spatial scale is in arcsec and the intensity scale indicates the relative intensity. We clearly see the central star and two dusty detached shells.

of precipitable water vapor in the atmosphere). Because the quality of mid-infared data is mostly affected by humidity, we obtained diffraction-limited images with a great stability (seeing+sky background) throughout the whole observating run. The images were deconvolved using the standard star HD 163376 as a measure of the point spread function (PSF, observed before and after the science target with the same integration time of $30 \mathrm{~s}$ ) and a maximum-of-likelihood algorithm with 50 iterations. Figure 1 shows the raw and deconvolved images in the three filters.

We obtained an optical spectrum of IRAS 17163 using the HERMES instrument (High Efficiency and Resolution Mercator Echelle Spectrograph; Raskin et al. 2011) on the Mercator telescope $(1.2 \mathrm{~m})$ in La Palma. HERMES is a high-resolution fiber-fed echelle spectrograph offering a spectral resolution $R \sim 80000$ and a 2.54 arcsec field of view. We obtained four exposures of $1200 \mathrm{~s}$ each, under mediocre seeing conditions, resulting in a signal-to-noise ratio of 20 at $6000 \AA$. The pipelinereduced spectrum stretches from 3770 to $9900 \AA$, but because of the red nature of the source, it only appears above the noise from $5300 \AA$ A onwards.

\section{A new post-red supergiant star}

Diffuse interstellar bands (DIBs), which are caused by absorption by the interstellar medium, are seen in our optical spectrum of IRAS 17163. This allows us to estimate the distance to the observed object.

We adopted the relation defined by Reid et al. (2009) to calculate the kinematic distances for interstellar clouds observed in the line-of-sight towards IRAS 17163. The KI doublet velocity profile is plotted in Fig. 2. Three main interstellar velocity components are observed at local standard of rest (LSR) velocities of $-31.7,-10.1$, and $5.9 \mathrm{~km} \mathrm{~s}^{-1}$. A possible weak component at $-58.5 \mathrm{~km} \mathrm{~s}^{-1}$ is visible in both KI profiles. In addition, we observe narrow $(F W H M \sim 0.15 \AA)$ interstellar $\mathrm{C}_{2}$ absorption lines at an LSR velocity of $-32.3 \mathrm{~km} \mathrm{~s}^{-1}$.

For the interstellar cloud at $\sim-32 \mathrm{~km} \mathrm{~s}^{-1}$ we obtain the strongest constraints on the kinematic distance. We find a lower limit of $3.6 \mathrm{kpc}$ for this interstellar cloud, which is also a lower limit of the distance to IRAS 17163.

If the Galactic rotation model is applied to the component at $5.9 \mathrm{~km} \mathrm{~s}^{-1}$, this would give a lower limit of $17.4_{-1.33}^{+1.84} \mathrm{kpc}$ to the star. This, however, is incompatible with the inferred total line-of-sight reddening. We consider it most likely that this diffuse cloudlet has a peculiar velocity with respect to the rotational/radial velocity of the Galactic disk in this direction. The weak $\mathrm{K}_{\mathrm{I}}$ cloud at $-58.5 \mathrm{~km} \mathrm{~s}^{-1}$ would correspond to a minimum distance of $4.7 \mathrm{kpc}$.

The $\mathrm{K}_{\mathrm{I}}$ velocity absorption profile is shown together with both the Galactic radial velocity (in LSR) and the visual extinction $A_{\mathrm{V}}$ as a function of distance.

From the measured equivalent widths for the 5780, 5797, 6379, and $6613 \AA$ DIBs we infer a reddening, $E(B-V)$, between 1.6 and 2.8 mag (Luna et al. 2008), with a mean of 2.1 mag (Table 1). The total-to-selective visual extinction for typical ISM dust is $R_{\mathrm{V}}=3.1$ (Fitzpatrick \& Massa 2009) and leads to a visual extinction of $6.4 \mathrm{mag}$. The maximum value for the reddening corresponds to $A_{\mathrm{V}} \sim 8.7 \mathrm{mag}$. In Fig. 2 we plot the visual extinction versus distance extracted from the 3D Galactic dust extinction map constructed by Drimmel et al. (2003). We also indicate the distances derived from interstellar visual extinction values of 6 and 9 mag towards IRAS 17163. Although the Galactic dust extinction model may not be entirely accurate for sightlines within 20 degrees towards the Galactic center, in this case the different distance determinations are fully consistent with each other.

In conclusion, the strong interstellar component at $-32 \mathrm{~km} \mathrm{~s}^{-1}$ and the observed visual extinction, $A_{\mathrm{V}} \geq 6 \mathrm{mag}$, suggests a distance of $3.6 \mathrm{kpc}$ to this IS cloud, and provides a lower limit to the distance of IRAS 17163. The maximum visual extinction ( $\sim 9 \mathrm{mag}$ ) consistent with the measured DIBs strengths implies an upper limit to the distance of $\sim 4.7 \mathrm{kpc}$. Therefore, the distance to IRAS 17163 is between 3.6 and $4.7 \mathrm{kpc}$.

This is four times farther than what was previously assumed by Le Bertre et al. (1989), who classified this star as a postAGB star. The luminosity of IRAS $17163\left(5 \times 10^{5} L_{\odot}\right.$, see Sect. 5.1) is thus well above the maximum luminosity of a postAGB star $\left(\sim 10^{4} L_{\odot}\right.$; Schoenberner et al. 1983). IRAS 17163 is clearly not a post-AGB star, but very likely a post-red supergiant. 


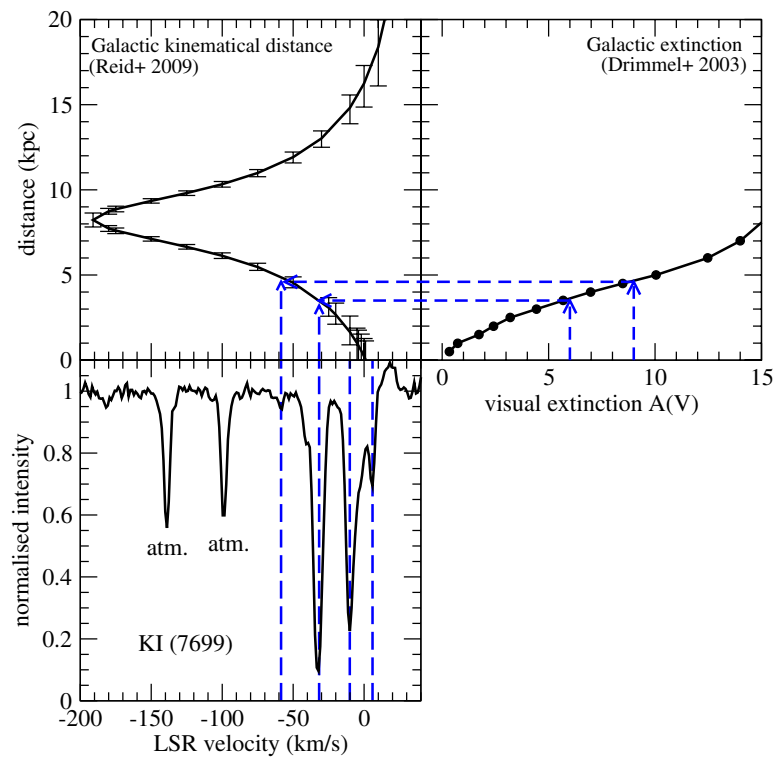

Fig. 2. K I doublet velocity profile (lower left) of IRAS 17163, together with the Galactic kinematical distance estimate (upper left) from Reid et al. (2009) and the model of Galactic extinction (upper right) from Drimmel et al. (2003). This indicates that the distance of IRAS 17163 is between 3.6 and $4.7 \mathrm{kpc}$.

Table 1. Measured DIB strengths (in $\AA$, with $10 \%$ uncertainties) towards IRAS 17163.

\begin{tabular}{cccc}
\hline \hline DIB & $E W(\AA)$ & $E W / E(B-V)$ & $E(B-V)$ for IRAS 17163 \\
\hline 5780 & 0.91 & 0.46 & 1.98 \\
5797 & 0.48 & 0.17 & 2.82 \\
6379 & 0.14 & 0.088 & 1.59 \\
6613 & 0.404 & 0.21 & 1.92 \\
8621 & 0.497 & 0.37 & 1.34 \\
\hline
\end{tabular}

Notes. The $8621 \AA$ DIB appears blended with a $\mathrm{H}_{\mathrm{I}}$ emission line and was therefore rejected for the calculation of $E(B-V)$. $E W / E(B-V)$ are taken from Luna et al. (2008).

\section{The double dust shell}

Le Bertre et al. (1989) obtained observations of IRAS 17163 and concluded that IRAS 17163 was located at $1 \mathrm{kpc}$ from the Sun, with a luminosity well below the maximum for post-AGB stars. The high polarization ( $p \sim 12 \%$ ) measured in $V, R$ and $I$ seems to indicate that the circumstellar envelope is highly aspherical. IRAS 17163 is unresolved in the optical images obtained with the ESO 1.5 m Danish telescope at la Silla (Chile), (Le Bertre et al. 1989), while infrared speckle interferometric observations indicate an angular dimension of $1.11 \pm 0.23^{\prime \prime}$ in $L$ band $(3.6 \mu \mathrm{m})$ (Starck et al. 1994). The high-resolution $\mathrm{H}_{\alpha}$ profile of IRAS 17163 indicates a wind velocity of $\sim 63 \mathrm{~km} \mathrm{~s}^{-1}$.

IRAS 17163 was observed by the HST in the optical (Siódmiak et al. 2008), but was classified as not resolved. The integration time was short (45 s) and after an inspection of the HST image, it appears that the dimension of the detached shells corresponds to the Airy pattern of the HST at the observed wavelengths, which is why the authors did not see the detached shells (Siódmiak, priv. comm.). The images of IRAS 17163 we obtained with VISIR are displayed Fig. 1. The object is clearly resolved in all filters, with a diameter of $\sim 5^{\prime \prime}$. These are the first direct images of the circumstellar material around this very bright infrared source. The mid-infrared morphology of IRAS 17163 is

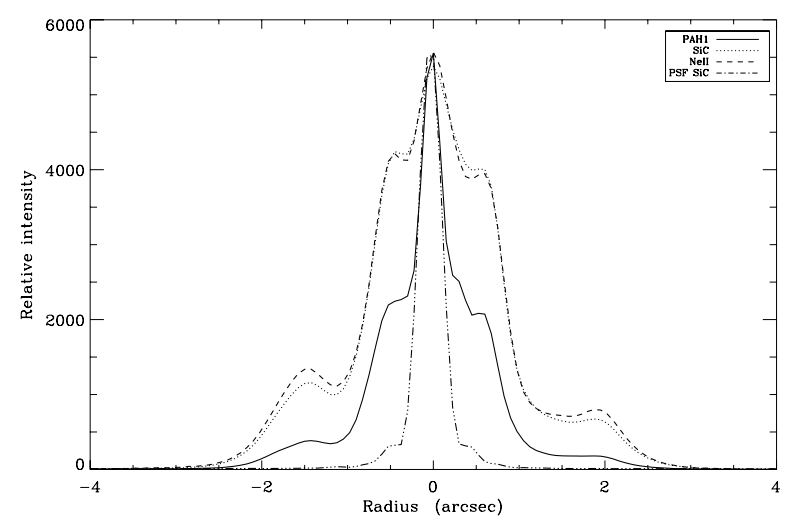

Fig. 3. Radial profile of the non deconvolved images in the three VISIR filters, showing the star and a double detached shell. The PSF is also shown for reference.

similar to a fried egg, and we dubbed it the "Fried Egg" nebula. The object is circular at large-scale and the central star can be observed in all filters.

The most striking aspect of these images is the presence of a double detached shell around the central star. This is more clearly visible in Fig. 3, which shows a cut along the north-south direction in the three deconvolved images. We can clearly see two shells at $\sim 0.6$ and $1.5^{\prime \prime}$ from the central star. The images show that those shells are quite round. However, the shells show some clumpy structures that are apparent in all images and are consequently not deconvolution artifacts. While on the raw images the shells seems to be very smooth, the deconvolved images clearly show that these shells are made of a large number of clumps. We can also see a large hole in the second dusty shell along a PA of $\sim 45^{\circ}$. An extended structure, which seems to link the central star and the first shell is also seen along a PA $\sim 170^{\circ}$. There are some signs for a departure from spherical symmetry in the core of the nebula.

Considering a distance to the object of $4 \mathrm{kpc}$, the two shells have a radius of 2400 and 6000 AU, respectively. Assuming a distance of $4 \mathrm{kpc}$ and an expansion velocity of $40 \mathrm{~km} \mathrm{~s}^{-1}$, this leads to an estimate of $\sim 435$ years for the time interval between the two shells.

To estimate the amount of dust present in the observed shells, we developed a radiative transfer model. We used the code described by Szczerba et al. (1997). The aim of our model is to fit both the SED and the intensity profile in our three VISIR images to get an estimate of the mass of dust in the envelope. The IRAS spectrum of IRAS 17163 shows silicate features, indicating that there is O-rich dust in the circumstellar envelope. Assuming a distance of $4 \mathrm{kpc}$, a star effective temperature of $8000 \mathrm{~K}$ and that the dust is made of circumstellar silicates with a standard MRN size distribution (Mathis et al. 1997), we obtained the best fit to the SED and radial dust distribution with a total dust mass of $0.04 M_{\odot}$, and a mass-loss rate varying with $r^{-1}$ between the shell, because we were unable to fit the SED without the presence of dust in between the shells. Assuming a lower limit of 100 for the gas-to-dust mass ratio, this leads to a total gas mass of $4 M_{\odot}$ in the ejecta and an average mass-loss rate of $10^{-4} M_{\odot} \mathrm{yr}^{-1}$ and 2-3 $\times 10^{-3} M_{\odot} \mathrm{yr}^{-1}$ associated with the formation of the inner and outer shells, respectively. Some dust is present in the intershell, and the presence of hot dust $(\sim 1500 \mathrm{~K})$ close to the central has to be assumed to reproduce the flux of the central unresolved source. In the first shell the mean dust temperature ranges from 230 to $180 \mathrm{~K}$, while in the outer shell it ranges from 150 to about $110 \mathrm{~K}$. Assuming a width of 0.5 arcsec for each shell, we 


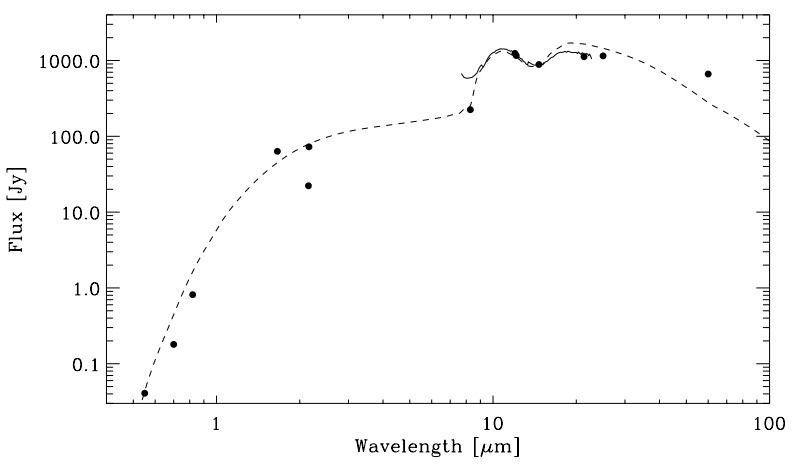

Fig. 4. Spectral energy distribution of IRAS 17163, the data points are from Szczerba et al. (2007). The dashed line represents our best radiative transfer model fit to the data.

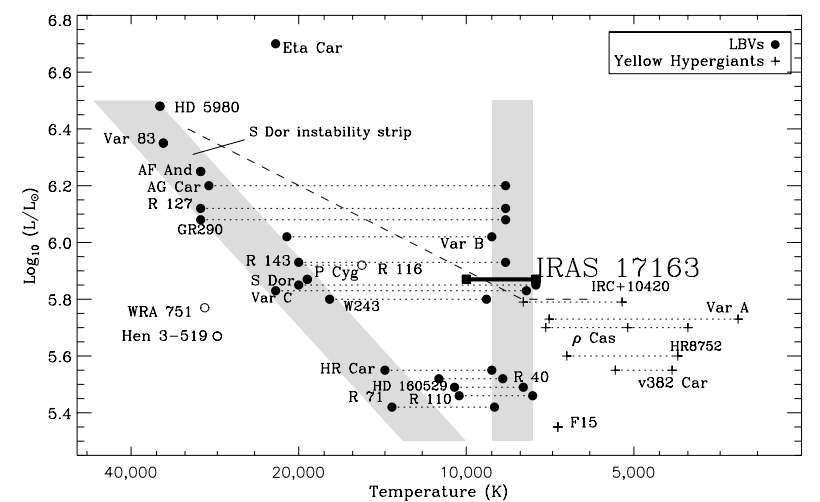

Fig. 5. Temperature-luminosity diagram of post-red supergiants, showing that IRAS 17163 likely is a yellow hypergiant. The grey bands on the left and right of the diagram represent respectively the regimes in the HR diagram that the LBVs occupy when they are in the quiescent phase (left) and the active phase (right) at maximum visual brightness (Smith et al. 2004).

found that 0.002 and $0.005 M_{\odot}$ of dust are present in the inner and outer shells respectively.

\section{Discussion}

\subsection{A yellow hypergiant}

The optical spectrum of IRAS 17163 shows the $7450 \AA$ NI line and no helium lines, which indicates that the central star is either a late B or early A central star (Gray \& Corbally 2009), with an effective temperature in the range $7500-10000 \mathrm{~K}$. If we assume, as estimated by the DIBs and the K I radial velocity we observed (Sect. 3), a distance of $4 \mathrm{kpc}$ for the object, its luminosity (measured by integrating the SED, Fig. 4 ) is $\sim 5 \times 10^{5} L_{\odot}$. If one compares the location of IRAS 17163 on a temperatureluminosity diagram (Fig. 5), as the one presented by Oudmaijer et al. (2009), we can see that its properties are very similar to the brightest yellow hypergiants like the famous IRC +10420 , with its temperature and luminosity just below the LVBs.

\subsection{Mass-loss variations}

Yellow hypergiants are not in a quiet evolutionary phase (Oudmaijer 2009) and display episodic ejections, moving alternatively from the blue to the red on an HR diagram. The two detached shells we observe are likely caused by such events.
HST imaging of the yellow hypergiant IRC +10420 has shown the presence of partial shell and knot structures, which represent episodic ejection events (Tiffany et al. 2010, and references therein). We observed two shells due to phases of enhanced mass-loss separated by $\sim 400$ years. The central stellar source in the 10-micron image cannot be fitted with a stellar SED, which could be due to hot dust close to the star, indicating a third, unresolved shell next to the central star. The presence of clumps of dust close to the central star could explain the polarization observed for IRAS 17163. The unusual high linear polarization could also be partly due to an alignment of interstellar grains in this line of sight. Polarization measurements of field stars near IRAS 17163 would be needed to resolve this question. Possibly there are many other shells farther away from the central star. These shells are not observed either because of the narrow field of view of VISIR, the fact that these shells would be less dense and thus weaker and/or the fact that dust further away would be colder and thus emit at longer wavelengths. These shells would explain the discrepancy between our radiative transfer model and the observed flux at 60 microns. Millimeter observations of the Fried Egg should allow the detection of these shells.

\section{Conclusions}

We presented the first resolved images of IRAS 17163. The morphology we observed led us to dub this object the "Fried Egg" nebula. We resolved two large concentric spherical dusty shells around the central star, which is itself embedded in dust. More than $4 M_{\odot}$ of gas and dust have already been ejected by the star. IRAS 17163 was previously classified as a post-AGB star, but the presence of DIBs in the optical spectra we obtained led us to the conclusion that the object is four times farther than previously suggested and is consequently a post-red supergiant. The location of the Fried Egg on an HR diagram and the remarkable similarity between its optical spectra and that of the prototype Yellow hypergiant IRC +10420 indicate that the Fried Egg probably is a yellow hypergiant. The detached shells around IRAS 17163 are a confirmation that non-quiescent mass-loss occurs during this phase of stellar evolution.

Acknowledgements. The authors would like to thank the anonymous referee for providing them with constructive comments and suggestions.

\section{References}

Drimmel, R., Cabrera-Lavers, A., \& López-Corredoira, M. 2003, A\&A, 409, 205 Epchtein, N., Le Bertre, T., Lepine, J. R. D., et al. 1987, A\&AS, 71, 39

Fitzpatrick, E. L., \& Massa, D. 2009, ApJ, 699, 1209

Gray, R. O., \& Corbally, C. J. 2009 (Princeton University Press)

Henize, K. G. 1976, ApJS, 30, 491

Lagadec, E., Verhoelst, T., Mekarnia, D., et al. 2011, MNRAS, 417, 32

Lagage, P. O., Pel, J. W., Authier, M., et al. 2004, The Messenger, 117, 12

Le Bertre, T., Heydari-Malayeri, M., Epchtein, N., Gouiffes, C., \& Perrier, C. 1989, A\&A, 225, 417

Luna, R., Cox, N. L. J., Satorre, M. A., et al. 2008, A\&A, 480, 133

Mathis, J. S., Rumpl, W., \& Nordsieck, K. H. 1977, ApJ, 217, 425

Oudmaijer, R. D., Davies, B., de Wit, W.-J., \& Patel, M. 2009, The Biggest, Baddest, Coolest Stars, 412, 17

Raskin, G., van Winckel, H., Hensberge, H., et al. 2011, A\&A, 526, A69

Reid, M. J., Menten, K. M., Zheng, X. W., et al. 2009, ApJ, 700, 137

Schoenberner, D. 1983, ApJ, 272, 708

Siódmiak, N., Meixner, M., Ueta, T., et al. 2008, ApJ, 677, 382

Smith, N., Vink, J. S., \& de Koter, A. 2004, ApJ, 615, 475

Starck, J.-L., Bijaoui, A., Lopez, B., \& Perrier, C. 1994, A\&A, 283, 349

Szczerba, R., Omont, A., Volk, K., Cox, P., \& Kwok, S. 1997, A\&A, 317, 859

Szczerba, R., Siódmiak, N., Stasińska, G., \& Borkowski, J. 2007, A\&A, 469, 799

Tiffany, C., Humphreys, R. M., Jones, T. J., \& Davidson, K. 2010, AJ, 140, 339 